“C 2016 IEEE. Personal use of this material is permitted. Permission from IEEE must be obtained for all other uses, in any current or future media, including reprinting/republishing this material for advertising or promotional purposes, creating new collective works, for resale or redistribution to servers or lists, or reuse of any copyrighted component of this work in other works." 


\title{
Evolutionary Lazy Learning for Naive Bayes Classification
}

\author{
Yu Bai*, Haishuai Wang*, Jia Wu*, Yun Zhang ${ }^{\dagger}$, Jing Jiang*, Guodong Long* \\ ${ }^{*}$ Centre for Quantum Computation \& Intelligent Systems (QCIS), \\ Faculty of Engineering and Information Technology, University of Technology Sydney, Australia \\ ${ }^{\dagger}$ School of Computer Science and Engineering, University of New South Wales, Australia \\ Email:Yu.Bai@uts.edu.au;Jia.Wu@uts.edu.au; Haishuai.Wang@uts.edu.au; \\ Zhangyuny@gmail.com; Guodong.Long@uts.edu.au; Jing.Jiang@uts.edu.au
}

\begin{abstract}
Most improvements for Naive Bayes (NB) have a common yet important flaw - these algorithms split the modeling of the classifier into two separate stages - the stage of preprocessing (e.g., feature selection and data expansion) and the stage of building the NB classifier. The first stage does not take the NB's objective function into consideration, so the performance of the classification cannot be guaranteed. Motivated by these facts and aiming to improve NB with accurate classification, we present a new learning algorithm called Evolutionary Local Instance Weighted Naive Bayes or ELWNB, to extend NB for classification. ELWNB combines local NB, instance weighted dataset extension and evolutionary algorithms seamlessly. Experiments on 20 UCI benchmark datasets demonstrate that ELWNB significantly outperforms NB and several other improved NB algorithms.
\end{abstract}

\section{INTRODUCTION}

Naive Bayes (NB), which is based on probability theory, is one of the most widely used learning algorithms [1], [2]. NB is computationally highly efficient and thus is suitable for many learning scenarios such as text classification [3], web mining [4], sentiment analysis [5] and image classification [6], [7]. Indeed, NB is a special case of Bayesian network [8], which considers the dependence of attributes to obtain the correct classification. The time consumption of the general Bayesian network can be very high, which affects its application in the real world. To address this issue, NB model adopts a simple and straightforward assumption on the general Bayesian network, i.e., the attributes of the training dataset are independent of each other. Under this assumption, the examination of dependence between attributes is no longer needed and building the NB model only needs linear mathematical computation.

In many real world applications, there is a strong dependence between attributes, the performance of the NB classification can drop sharply. Furthermore, NB treats the different instances with the same weight, but training instances that are closer to the test instance may play a more important role than those that are far from the test instance. For this reason, many approaches have attempted to improve the performance of NB [9], including feature based learning
[10], [11], [12], [13], [14], structure extension based learning [15], [16], [17], local learning [18] and data expansion based learning [19], [20].

The above methods of improving NB have achieved good results, however, after carefully investigation, we find that all these methods have a common disadvantage (i.e., the NB models are separated into two steps). The first stage is the preprocessing, and the second is the creation of the NB model. These two stages are independent of each other and the first stage is carried out without consideration of the NB objective function. As a result, the performance of the NB cannot be guaranteed.

To solve the common disadvantage of the above methods, we propose a new method in this paper called Evolutionary Local Instance Weighted Naive Bayes, namely ELWNB. ELWNB constructs an extended training dataset based on the original training dataset using instances cloning, which will be used to build a NB classifier for further classification. Indeed, how the extended training dataset is constructed is determined by two important parameters: threshold (determines whether this instance should be cloned) and weight (determines the number of times that this instance should be cloned). Here, the Differential Evolution (DE) algorithm [21] is used to automatically search for the optimal parameters. To obtain the best classification result, the NB's objective function is directly used as the fitness function of the DE to evaluate the performance of different combinations of the above two parameters. Experiments and comparisons on $20 \mathrm{UCI}$ benchmark datasets demonstrate the performance of proposed ELWNB.

\section{EVOLUTIONARY LAZY LEARNING FOR NB}

\section{A. EWLNB Algorithm}

First, we define the distance of two instances as

$$
d(m, n)=\sqrt{\sum_{i=1}^{k}\left(m_{i}-n_{i}\right)^{2}}
$$




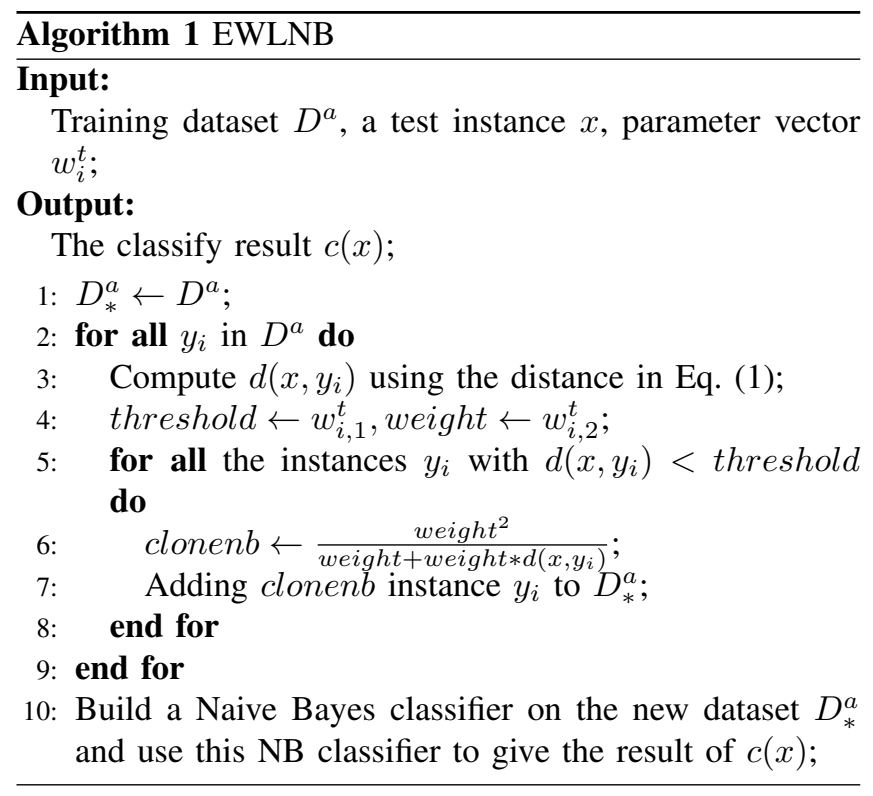

where $m$ and $n$ are two vectors in a K-dimension space with the value $\left\{m_{1}, \cdots m_{k}\right\}$ and $\left\{n_{1}, \cdots n_{k}\right\}$, respectively.

Given a training dataset $D^{a}$ and a test instance $x$, we first use Eq. (1) to calculate the distance $d\left(x, y_{i}\right)$ between the test instance $x$ and each training instance $y_{i}$ in $D^{a}$. Then, if $d\left(x, y_{i}\right)$ satisfies the requirements of the parameter threshold, we get the clonen $b$ of the $i$ th individual through $d\left(x, y_{i}\right)$, and add clonenb clones of $y_{i}$ into $D_{*}^{a}$ to expand the training dataset $D^{a}$. We deploy a NB classifier on the expanded $D_{*}^{a}$ to calculate the class classification $c(x)$. The detailed EWLNB is depicted in Algorithm 1.

Here $D_{*}^{a}$ is the extended training dataset, $w_{i, 1}^{t}$ and $w_{i, 2}^{t}$ are the two dimensions of the parameter vector, in our algorithm, they represent threshold and weight respectively. clonenb is a integer which represents the number of times that should be cloned of the training instance $y_{i}$.

It is clear that EWLNB algorithm's main procedure is expanding the training instances $D^{a}$ for a test instance $x$. We call our learning algorithm Evolutionary Local Instance Weighted Naive Bayes, or EWLNB, because it spends no effort during training time, delays all computation until classification time and the evolutionary algorithm is used to achieve the optimal parameter vector. Our learning algorithm deals with NB's shortcomings by cloning some of the training instances to produce an expanded training dataset.

\section{B. Evolution of the Parameter threshold and weight}

To obtain the optimal parameters to get the extended dataset $D_{*}^{a}$, we use DE to learn two important parameters for EWLNB classification. In our solution, the different combinations of the two parameters act as candidates, presented by parameter vector $W$. The main stages of the evolution are initialization, cloning, mutation, and selection. The evolving optimization will assist the discovery of the optimal $W$ vector with the best classification accuracy. Before introducing details of the algorithm, we define a number of notations.

- $W$ represents the set of candidates, which we call the population. $W=\left\{w_{1}, \cdots, w_{L}\right\}$ where $L$ represents the size of the population, $w_{i}=\left\{w_{i, 1}, \cdots, w_{i, n}\right\}$ represents a single candidate, which we call an individual, and where $n$ is the size of the parameter vector. In our algorithm, the value of $n$ is two.

- $w_{i, j}$ denotes the $j$ th value of the $i$ th individual.

- $w_{c}$ represents the individual which has the best fitness (i.e.,best classification accuracy) on the test instances.

The detailed DE process is descripted as following steps:

1) Initialization of the Parameter Vectors: For individuals in $W=\left\{w_{1}, \cdots, w_{L}\right\}$ with a population size $L$, we should ensure that every individual $w_{i}=\left\{w_{i, 1}, \cdots, w_{i, n}\right\}$ in the population is generated through certain random mechanisms. We therefore set $w_{i}^{j}$ value of $w_{i}$ for each individual as a uniformly distributed random number within the range $(0$, 1]. In the experiment, $90 \%$ of the original training instances $D$ are used as the training dataset $D^{a}$ to learn $w_{c}$ and the remaining instances are used as the test dataset $D^{b}$, with the population size $L$ set to 50 .

2) Evaluation of EWLNB: The process of Evaluation of EWLNB can be divided into following steps:

Calculation of Fitness Function: the fitness of the $i$ th individual of the $t$ th generation is the classification accuracy that is obtained by EWLNB using the $w_{i}^{t}$ to carry out the probability estimation. The calculation of fitness function can be described as:

$$
f\left[w_{i}^{t}\right]=\frac{1}{N^{b}} \sum_{1}^{N^{b}} \delta\left[c\left(x_{i}^{b}\right), y_{i}^{b}\right]
$$

where $c\left(x_{i}^{b}\right)$ is the classification result of the $i$ th instance in test dataset $D^{b}$ with $N^{b}$ instances, using Algorithm 1 based on individual $w_{i}^{t}$. $y_{i}^{b}$ is the actual class value of the $i$ th instance. $\delta\left[c\left(x_{i}^{b}\right), y_{i}^{b}\right]$ is one if $c\left(x_{i}^{b}\right)=y_{i}^{b}$ and zero otherwise.

Individual Selection: we sort the individuals in every population according to the fitness of each individual, and choose the individual $w_{c}^{t}$ with the best fitness performance in the $t$ th generation to use as the base of the DE mutation.

Individual Mutation: we use the mutate operation to get the mutation individuals in the $t$ th generation through small changes over the best individual of the current generation. For any individual $w_{i}^{t}$ from the $t$ th generation, the new variation individual $v_{i}^{t}$ can be generated as followings.

$$
v_{i}^{t}=w_{c}^{t}+F *\left(w_{i}^{t}-w_{j}^{t}\right)
$$

where $w_{i}^{t}$ and $w_{j}^{t}$ are two different individuals of generation $t, F$ is a normally distributed random variable within the range $[0,1]$. 


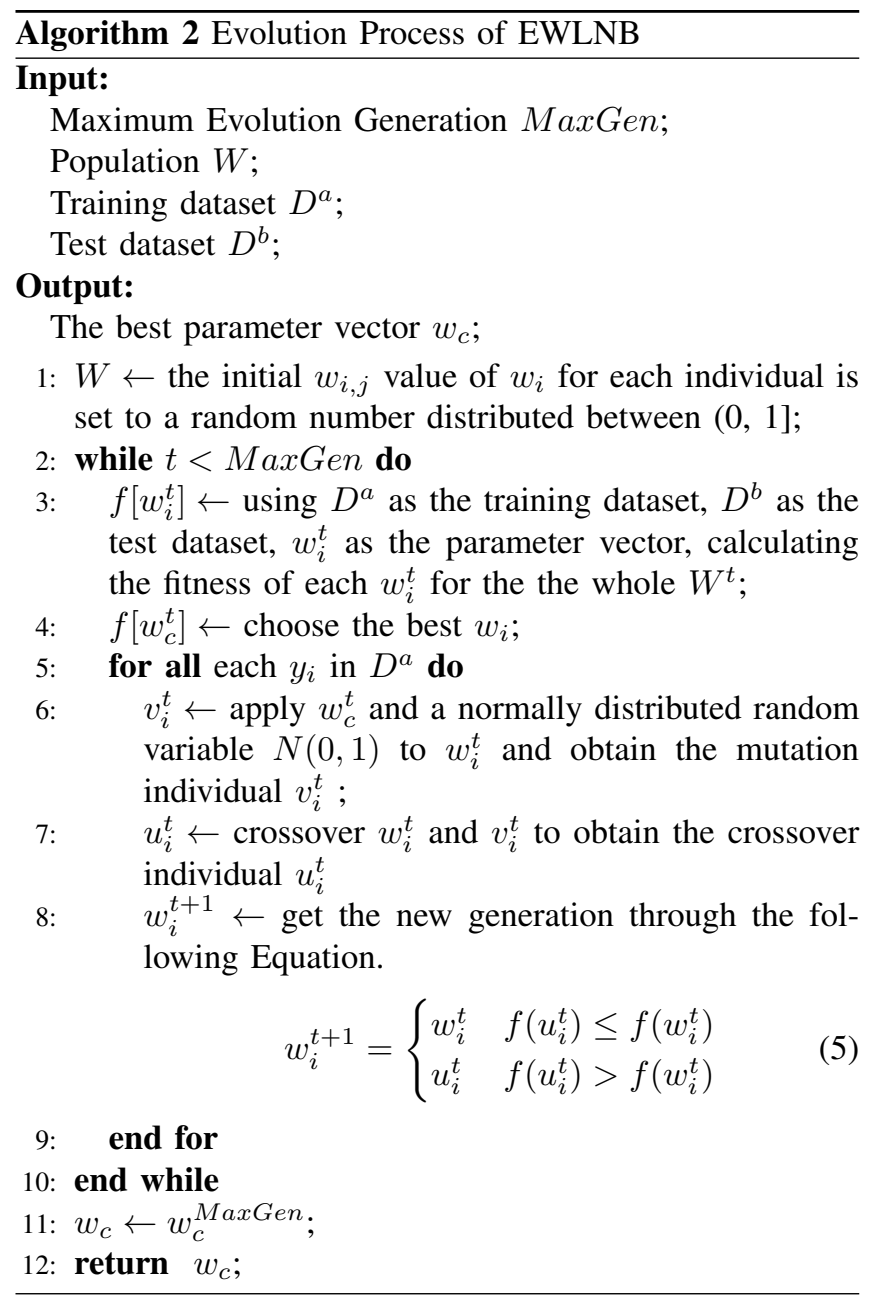

Individual Crossover: we use the crossover operation to exchange the dimensions of vectors in the $t$ th generation of $W^{t}$ and $V^{t}$. For each $w_{i}^{t}$ in $W^{t}$, there is a corresponding $v_{i}^{t}$ in $V^{t}$. The crossover vectors are created through $W^{t}$ and $V^{t}$ to involve more varieties using the following equation.

$$
u_{j, i}^{t}= \begin{cases}v_{j, i}^{t} & \text { if }\left(\operatorname{rand}_{j, i} \leq C R\right) \operatorname{or}\left(j=j_{\text {rand }}\right) \\ w_{j, i}^{t} & \text { otherwise }\end{cases}
$$

where $w_{j, i}^{t}$ is the $j$ th dimension of the $i$ th individual in the $t$ th generation. $u_{j, i}^{t}$ is the $j$ th dimension of the $i$ th mutation vector in the $t$ th generation, $v_{j, i}^{t}$ is the formed $j$ th dimension of the $i$ th crossover vector in the $t$ th generation. $C R$ is a fixed parameter for the whole algorithm within the range $[0,1]$, and in our algorithm, the value of $C R$ is 0.5 . $\operatorname{rand}_{j, i}$ is a normally distributed random variable within the range $[0,1]$, which is generated for every dimension of the vector, and $j_{\text {rand }}$ is a normally distributed random integer within the range $[0, n]$, where $n$ is the dimension of the individual. In our algorithm, the value of $n$ is two, $j_{\text {rand }}$ is generated once for every individual.
3) Update of EWLNB: To determine whether the crossover individual $u_{i}^{t}$ can replace the target individual vector $w_{i}^{t}$ to be the new individual $w_{i}^{t+1}$ in the $t+1$ th generation, the EWLNB algorithm uses Eq. (5) to adopt a greedy search strategy. It is chosen as the offspring only if the fitness of $u_{i}^{t}$ is better than that of the target individual $w_{i}^{t}$, otherwise, the individual $w_{i}^{t}$ is maintained in the $t$ th generation. Following this process, the system again chooses the individual $w_{c}^{t+1}$ with the best fitness performance in the $t+1$ th generation as the new local optimal individual.

A complete evolutionary process (as shown in Algorithm 2) of the population includes Evaluation and Update, which continuously repeats until the algorithm surpasses the pre-set maximum number $\operatorname{MaxGen}$, or the same result is obtained for a number of consecutive iterations. After obtaining the best individual $w_{c}$, corresponding to the obtained parameter values of threshold and weight, we use the values to construct the extended Training dataset $D^{*}$ and build a NB classifier over $D^{*}$ to classify the test instances.

\section{EXPERIMENT}

\section{A. Benchmark data and parameters}

We implement the proposed method using the WEKA [22], [23] data mining tool and validate its performance on 20 benchmark datasets from the UCI data repository [24]. The detailed information of the 20 datasets is shown through table I. Because NB is designed for categorical attributes, we first replace all missing attribute values in our experiment using the unsupervised attribute filter ReplaceMissingValues in WEKA. Then, we apply unsupervised filter Discretize in WEKA to discretize numeric attributes into nominal attributes. The three parameters $L$, MaxGen and $C R$ in our algorithm are set to 50, 50, and 0.5, respectively. All results are obtained via 10 runs of 10 -fold cross validation.

\section{B. Baseline Methods}

For comparison purposes, we compare ELWNB with the following baseline methods.

- NB: a standard Naive Bayes classifier with conditional attribute independence assumption.

- LNB: a lazy Naive Bayes classifier which calculates the distances of instances through attribute similarity.

\section{Evaluation criterion}

In our experiments, the selected algorithms are evaluated on the criterion of classification accuracy (measured by ACC), which is calculated by the percentage of successful predictions on domain specific problems [25], [26], [27]. The detailed experiment results are shown in Table II and table III. We compare our algorithm with each other algorithm via a two-tailed t-test with significantly different probability of 0.95 , because we speak of two results for a dataset as being "significantly different" only if the difference is statistically 
Table I

20 UCI DATASETS.

\begin{tabular}{|c|c|c|c|c|c|}
\hline Datasets & Instances & Attributes & Classes & Missing & Numeric \\
\hline anneal & 898 & 39 & 6 & $\mathrm{Y}$ & $\mathrm{Y}$ \\
\hline lymph & 148 & 19 & 4 & $\mathrm{~N}$ & $\mathrm{Y}$ \\
\hline artificial & 10218 & 8 & 10 & $\mathrm{~N}$ & $\mathrm{~N}$ \\
\hline monks & 556 & 7 & 2 & $\mathrm{~N}$ & $\mathrm{~N}$ \\
\hline audiology & 226 & 70 & 24 & $\mathrm{Y}$ & $\mathrm{N}$ \\
\hline newthyroid & 215 & 6 & 3 & $\mathrm{~N}$ & $\mathrm{~N}$ \\
\hline energy & 768 & 9 & 37 & $\mathrm{~N}$ & $\mathrm{~N}$ \\
\hline primary-tumor & 339 & 18 & 21 & $\mathrm{Y}$ & $\mathrm{N}$ \\
\hline glass & 214 & 10 & 7 & $\mathrm{~N}$ & $\mathrm{Y}$ \\
\hline qar & 1055 & 42 & 2 & $\mathrm{~N}$ & $\mathrm{~N}$ \\
\hline hypothyroid & 3772 & 30 & 4 & $\mathrm{Y}$ & $\mathrm{Y}$ \\
\hline robot & 5456 & 25 & 4 & $\mathrm{~N}$ & $\mathrm{~N}$ \\
\hline ionosphere & 351 & 35 & 2 & $\mathrm{~N}$ & $\mathrm{Y}$ \\
\hline sick & 3772 & 30 & 2 & $\mathrm{Y}$ & $\mathrm{Y}$ \\
\hline iris & 150 & 5 & 3 & $\mathrm{~N}$ & $\mathrm{Y}$ \\
\hline vehicle & 846 & 19 & 4 & $\mathrm{~N}$ & $\mathrm{Y}$ \\
\hline labor & 57 & 17 & 2 & $\mathrm{Y}$ & $\mathrm{Y}$ \\
\hline vowel & 990 & 14 & 11 & $\mathrm{~N}$ & $\mathrm{Y}$ \\
\hline letter & 20000 & 17 & 26 & $\mathrm{~N}$ & $\mathrm{Y}$ \\
\hline zoo & 101 & 18 & 7 & $\mathrm{~N}$ & $\mathrm{Y}$ \\
\hline
\end{tabular}

significant at the 0.05 level according to the corrected twotailed t-test [28], as shown in Table II. Table III shows the results of the two-tailed t-test with a confidence level of 95\% between each pair of algorithms in terms of accuracy. Each entry $w / t / 1$ in the tables means that the algorithm in the corresponding row wins in $\mathrm{w}$ datasets, ties in $\mathrm{t}$ datasets, and loses in 1 datasets, compared to the algorithm in the corresponding column. The results displayed in Tables II and III show that ELWNB significantly outperforms LNB and NB measured by ACC. We summarize the highlights as

1. It is clear that ELWNB significantly outperforms NB measured by ACC: the w/t/l value on ACC between ELWNB and NB is $8 / 12 / 0$. The average ACC for ELWNB is $83.11 \%$, which is higher than the average ACC $76.23 \%$ of NB.

2. It can also be seen that ELWNB significantly outperforms LNB measured by ACC: the w/t/l value on
ACC between ELWNB and LNB is 8/12/0. The average ACC for ELWNB is $83.11 \%$, which is higher than the average ACC $80.25 \%$ of LNB.

\section{CONCLUSIONS}

In this paper, we first investigated the classification performance of improved NB methods and found that they all have a common disadvantage, that is, the building of the NB classifier is carried out without consideration of the NB's objective function. Motivated by this observation, we presented a new evolutionary lazy learning algorithm called ELWNB. We experimentally tested the proposed algorithm ELWNB, using 20 UCI datasets recommended by WEKA, and compared our algorithm ELWNB with NB and LNB. The experimental results show that our algorithm significantly outperforms NB and LNB in yielding accurate classification. We believe that our work provides an effective 
Table II

EXPERIMENTAL RESULTS: ACC AND STANDARD DEVIATION.

\begin{tabular}{|c|c|c|c|c|c|}
\hline Datasets & ELWNB & LNB & & NB & \\
\hline anneal & $97.77 \pm 1.82$ & $97.44 \pm 1.58$ & $\circ$ & $94.32 \pm 2.38$ & $\circ$ \\
\hline lymph & $84.33 \pm 8.16$ & $86.33 \pm 8.80$ & & $85.67 \pm 9.55$ & \\
\hline artificial & $68.58 \pm 1.84$ & $47.72 \pm 1.19$ & $\circ$ & $36.40 \pm 1.00$ & $\circ$ \\
\hline monks & $97.29 \pm 2.75$ & $75.36 \pm 2.53$ & $\circ$ & $74.64 \pm 2.15$ & $\circ$ \\
\hline audiology & $77.39 \pm 5.55$ & $78.32 \pm 7.12$ & & $71.23 \pm 7.03$ & \\
\hline newthyroid & $95.30 \pm 4.98$ & $92.99 \pm 5.08$ & & $92.08 \pm 4.46$ & \\
\hline energy & $65.76 \pm 4.86$ & $58.85 \pm 4.30$ & $\circ$ & $45.05 \pm 3.92$ & $\circ$ \\
\hline primary-tumor & $47.49 \pm 5.20$ & $47.50 \pm 4.90$ & & $46.89 \pm 4.32$ & \\
\hline glass & $58.40 \pm 7.05$ & $59.85 \pm 7.39$ & & $60.32 \pm 9.69$ & \\
\hline qar & $80.29 \pm 4.91$ & $81.05 \pm 3.37$ & & $79.81 \pm 4.43$ & \\
\hline hypothyroid & $92.82 \pm 1.37$ & $92.84 \pm 0.88$ & & $92.79 \pm 1.02$ & \\
\hline robot & $89.08 \pm 1.49$ & $83.84 \pm 1.79$ & $\circ$ & $80.57 \pm 2.02$ & $\circ$ \\
\hline ionosphere & $91.17 \pm 3.42$ & $91.44 \pm 3.82$ & & $90.89 \pm 3.49$ & \\
\hline sick & $97.64 \pm 0.63$ & $97.08 \pm 0.54$ & $\circ$ & $96.74 \pm 0.53$ & $\circ$ \\
\hline iris & $95.33 \pm 8.34$ & $96.67 \pm 4.71$ & & $94.67 \pm 8.20$ & \\
\hline vehicle & $64.18 \pm 4.54$ & $67.86 \pm 4.73$ & & $61.82 \pm 3.54$ & \\
\hline labor & $91.67 \pm 14.16$ & $90.00 \pm 14.05$ & & $93.33 \pm 11.65$ & \\
\hline vowel & $90.61 \pm 2.70$ & $87.68 \pm 2.22$ & $\circ$ & $67.07 \pm 4.21$ & $\circ$ \\
\hline letter & $80.95 \pm 2.30$ & $76.00 \pm 2.23$ & $\circ$ & $66.15 \pm 2.15$ & ○ \\
\hline zoo & $96.18 \pm 6.54$ & $96.18 \pm 6.54$ & & $94.18 \pm 6.60$ & \\
\hline AVERAGE & $83.11 \pm 4.60$ & $\mathbf{8 0 . 2 5} \pm \mathbf{3 . 8 9}$ & & $76.23 \pm 4.62$ & \\
\hline
\end{tabular}

०: Statistically significant degradation.

Table III SUMMARY OF TWO-TAILED T-TEST RESULTS

\begin{tabular}{ccc}
\hline & LNB & NB \\
\hline NB & $0 / 13 / 7$ & \\
\hline ELWNB & $8 / 12 / 0$ & $8 / 12 / 0$ \\
\hline
\end{tabular}

data mining classification algorithm. An aspect of ELWNB that could clearly be investigated further is the method of calculating the number of times that a specific instance should be copied. Extending ELWNB to calculate the times that a specific instance should be copied in a more efficient way is our main direction for future research. 


\section{ACKNOWLEDGMENTS}

This work was supported in part by the China Scholarship Council Foundation (No. 201206410056), and the Australian Research Council (ARC) Discovery Projects under Grant No. DP140100545.

\section{REFERENCES}

[1] N. Friedman, D. Geiger, and M. Goldszmidt, "Bayesian network classifiers," Machine Learning, vol. 29, no. 2, pp. 131-163, 1997.

[2] D. Berend and A. Kontorovich, "A finite sample analysis of the naive bayes classifier," Journal of Machine Learning Research, vol. 16, pp. 1519-1545, 2015.

[3] J. Chen, H. Huang, S. Tian, and Y. Qu, "Feature selection for text classification with naïve bayes," Expert Systems with Applications, vol. 36, no. 3, pp. 5432-5435, 2009.

[4] C. Zhang, G.-R. Xue, Y. Yu, and H. Zha, "Web-scale classification with naive bayes," in Proceedings of the 18th International Conference on World Wide Web $(W W W)$. ACM, 2009, pp. 1083-1084.

[5] P. Shanmuganathan and C. Sakthivel, "An efficient naive bayes classification for sentiment analysis on twitter," Data Mining and Knowledge Engineering, vol. 7, no. 5, pp. 180$185,2015$.

[6] R. Timofte, T. Tuytelaars, and L. Van Gool, "Naive bayes image classification: beyond nearest neighbors," in Proceedings of the 11th Asian Conference on Computer Vision (ACCV). Springer, 2012, pp. 689-703.

[7] S. McCann and D. G. Lowe, "Local naive bayes nearest neighbor for image classification," in Proceedings of the IEEE Conference on Computer Vision and Pattern Recognition (CVPR). IEEE, 2012, pp. 3650-3656.

[8] J. Hernández-González, I. Inza, and J. A. Lozano, "Learning bayesian network classifiers from label proportions," Pattern Recognition, vol. 46, no. 12, pp. 3425-3440, 2013.

[9] L. Jiang, D. Wang, Z. Cai, and X. Yan, "Survey of improving naive bayes for classification," in Advanced Data Mining and Applications. Springer, 2007, pp. 134-145.

[10] P. Langley and S. Sage, "Induction of selective bayesian classifiers," in Proceedings of the Tenth International Conference on Uncertainty in Artificial Intelligence (UAI). Morgan Kaufmann Publishers Inc., 1994, pp. 399-406.

[11] J. Wu and Z. Cai, "Attribute weighting via differential evolution algorithm for attribute weighted naive bayes (wnb)," Journal of Computational Information Systems, vol. 7, no. 5, pp. 1672-1679, 2011.

[12] N. A. Zaidi, J. Cerquides, M. J. Carman, and G. I. Webb, “Alleviating naive bayes attribute independence assumption by attribute weighting," Journal of Machine Learning Research, vol. 14, pp. 1947-1988, 2013.
[13] J. Wu, S. Pan, X. Zhu, Z. Cai, P. Zhang, and C. Zhang, "Selfadaptive attribute weighting for naive bayes classification," Expert Systems With Applications, vol. 42, no. 3, pp. 14871502, 2015.

[14] J. Wu and Z. Cai, "A naive bayes probability estimation model based on self-adaptive differential evolution," Journal of Intelligent Information Systems, vol. 42, no. 3, pp. 671694, 2014.

[15] C. Chow and C. Liu, "Approximating discrete probability distributions with dependence trees," IEEE Transactions on Information Theory, vol. 14, no. 3, pp. 462-467, 1968.

[16] L. Jiang, H. Zhang, and Z. Cai, "A novel bayes model: Hidden naive bayes," IEEE Transactions on Knowledge and Data Engineering, vol. 21, no. 10, pp. 1361-1371, 2009.

[17] J. Wu, S. Pan, X. Zhu, P. Zhang, and C. Zhang, "SODE self-adaptive one-dependence estimators for classification," Pattern Recognition, vol. 51, pp. 358-377, 2016.

[18] E. Frank, M. Hall, and B. Pfahringer, "Locally weighted naive bayes," in Proceedings of the Nineteenth International Conference on Uncertainty in Artificial Intelligence (UAI). Morgan Kaufmann Publishers Inc., 2002, pp. 249-256.

[19] L. Jiang and Y. Guo, "Learning lazy naive bayesian classifiers for ranking," in Proceedings of the 17th IEEE International Conference on Tools with Artificial Intelligence (ICTAI). IEEE, 2005, pp. 5-pp.

[20] J. Wu, S. Pan, Z. Cai, X. Zhu, and C. Zhang, "Dual instance and attribute weighting for naive bayes classification," in Proceedings of the International Joint Conference on Neural Networks (IJCNN). IEEE, 2014, pp. 1675-1679.

[21] K. V. Price, "Differential evolution vs. the functions of the 2 nd iceo," in IEEE International Conference on Evolutionary Computation (CEC). IEEE, 1997, pp. 153-157.

[22] I. H. Witten and E. Frank, Data Mining: Practical machine learning tools and techniques. Morgan Kaufmann, 2005.

[23] R. R. Bouckaert, E. Frank, M. A. Hall, G. Holmes, B. Pfahringer, P. Reutemann, and I. H. Witten, "Wekaexperiences with a java open-source project," The Journal of Machine Learning Research, vol. 11, pp. 2533-2541, 2010.

[24] A. Asuncion and D. Newman, "Uci machine learning repository," 2007.

[25] J. Wu, X. Zhu, C. Zhang, and P. S. Yu, "Bag constrained structure pattern mining for multi-graph classification," IEEE Transactions on Knowledge and Data Engineering, vol. 26, no. 10, pp. 2382-2396, 2014.

[26] J. Wu, S. Pan, X. Zhu, and Z. Cai, "Boosting for multi-graph classification," IEEE Transactions on Cybernetics, vol. 45, no. 3, pp. 416-429, 2015.

[27] J. Wu, S. Pan, X. Zhu, C. Zhang, and X. Wu, "Positive and unlabeled multi-graph learning," IEEE Transactions on Cybernetics, vol. PP, no. 99, pp. 1-12, 2016.

[28] C. Nadeau and Y. Bengio, "Inference for the generalization error," Machine Learning, vol. 52, no. 3, pp. 239-281, 2003. 